\title{
The Effect of Valgus Reduction on the Position of the Blade of the Proximal Femoral Nail Antirotation in Intertrochanteric Hip Fractures
}

\author{
Eui Yub Jung, MD, In Taek Oh, MD*, Sang Yeup Shim, MD*, Byung Ho Yoon, $\mathrm{MD}^{\dagger}$, Yerl Bo Sung, $\mathrm{MD}^{*}$ \\ Department of Orthopedic Surgery, Samsung Medical Center, Seoul, \\ ${ }^{*}$ Department of Orthopedic Surgery, Sanggye Paik Hospital, Inje University College of Medicine, Seoul, \\ ${ }^{\dagger}$ Department of Orthopedic Surgery, Seoul Paik Hospital, Inje University College of Medicine, Seoul, Korea
}

Background: The purpose of this study was to evaluate the quantitative association between the degree of reduction and the position of the blade of the proximal femoral nail antirotation (PFNA) in intertrochanteric hip fractures.

Methods: From March 2009 to April 2015, 530 patients treated with PFNA for intertrochanteric hip fractures were retrospectively reviewed. Patients were divided into a valgus reduced group (group 1) and a non-valgus reduced group (group 2), and the "valgus reduced" was defined as valgus reduction over $5^{\circ}$. We compared the calcar referenced tip-apex distance (calTAD) and the area between the blade of PFNA and the medial cortex of the femoral neck between the two groups.

Results: The calTAD was measured as $22.5 \pm 4.1 \mathrm{~mm}$ in group 1 and $24.8 \pm 3.8 \mathrm{~mm}$ in group $2(p<0.05)$. The area between the blade and the medial femoral neck was measured as $135.5 \pm 49.8 \mathrm{~mm}^{2}$ in group 1 and $145.1 \pm 54.8 \mathrm{~mm}^{2}$ in group 2 ( $\left.p=0.074\right)$. The area corrected for the length difference in the femoral neck was $0.55 \pm 0.16$ in group 1 and $0.79 \pm 0.19$ in group $2(p<0.05)$.

Conclusions: Valgus reduction resulted in less calTAD and inferior position of the blade at the femoral neck in the treatment of intertrochanteric hip fractures with PFNA.

Keywords: Femur, Intertrochanteric fractures, Intramedullary nailing, Fracture closed reduction

Intertrochanteric hip fractures in the elderly are an important orthopedic problem globally due to the aging population and the increasing incidence of osteoporosis. Intertrochanteric hip fractures have a high rate of fusion, but the subsequent functional outcome is poor, and the mortality rate within 1 year after the injury has been reported to be as high as $12 \%$ to $36 \% .^{1)}$ Surgical treatment is the most important method in the intertrochanteric hip fractures. The goal of the treatment is to restore preoperative function and mobility in a short time. As an operative treatment for intertrochanteric hip fractures, extramedul-

Received September 27, 2018; Accepted October 31, 2018

Correspondence to: Yerl Bo Sung, MD

Department of Orthopedic Surgery, Sanggye Paik Hospital, Inje University

College of Medicine, 1342 Dongil-ro, Nowon-gu, Seoul 01757, Korea

Tel: +82-2-950-1029, Fax: +82-2-950-4897

E-mail: ybs58@paik.ac.kr lary fixation and intramedullary fixation have been used. Among these, intramedullary fixation is increasingly used for biomechanical advantages and less invasiveness. ${ }^{2-4)}$

One of the most important factors in intramedullary fixation is the quality of reduction. In varus reduction, the force applied to the implant increases, and the stability of the fracture is decreased, leading to a loss of reduction. ${ }^{5}$ Therefore, many surgeons use valgus reduction to increase the compressive force between the bone fragments and reduce the force between the bones and the implant to prevent loss of reduction. ${ }^{6,7)}$ Another important factor in intramedullary fixation for intertrochanteric hip fractures is the position of the blade at the femoral head. ${ }^{8)}$ In this regard, attempts have been made to decrease the tip-apex distance (TAD), which emphasizes positioning as deeply as possible in the center of the femoral head. In recent years, the concept of calcar referenced tip-apex distance (calTAD) has emerged on the grounds that placing 
the blade in the inferior part of the femoral neck and head is more biomechanically and clinically advantageous. ${ }^{9-12)}$ Therefore, non-varus reduction and the good position of the blade, the two important factors in intramedullary fixation, should always be kept in mind during the surgery.

However, there is no study that explains the relationship between the status of reduction and the position of the blade. We hypothesized that the status of reduction would affect the position of the blade. The purpose of this study was to investigate the relationship between the status of reduction and the position of the blade when proximal femoral nail antirotation (PFNA) was used in intertrochanteric hip fractures.

\section{METHODS}

\section{Patients and Exclusions Criteria}

A total of 585 patients who were treated with PFNA among patients who visited Inje University Sanggye Paik Hospital for intertrochanteric hip fractures from March 2009 to April 2015 were retrospectively reviewed. We conducted this study in compliance with the principles of the Declaration of Helsinki. The protocol of this study was reviewed and approved by the Institutional Review Board of Sanggye Paik Hospital (IRB No. 2018-05-022).

Of these, 30 patients were excluded because they underwent arthroplasty or internal fixation for proximal femoral fractures and measurement of the contralateral neck-shaft angle were not available. In addition, 25 patients were excluded because the distance from the blade tip to the subchondral bone was more than $15 \mathrm{~mm}$ or less than $10 \mathrm{~mm}$. Ultimately 530 patients were included in this study. Their mean age was $78.7 \pm 10.6$ years, and there were 133 males and 397 females (Fig. 1).

\section{Surgical Technique}

All patients underwent surgery under epidural or general anesthesia. One single surgeon (YBS) performed the same valgus reduction procedure for all the patients to compare the neck shaft angle with that of the uninjured femur. First, external rotation of the affected leg was performed, followed by traction, abduction, adduction, and internal rotation in the described order. The reduction of the fracture site was confirmed by an image amplifier. ${ }^{13)}$

If the femoral head center was located higher than the greater trochanter tip in the preoperative radiographs, $130^{\circ}$ PFNA was used; if not, $125^{\circ}$ PFNA was used. The surgeon positioned the blade tip within $10-15 \mathrm{~mm}$ of the subchondral bone on the anteroposterior and lateral views of the image amplifier in all patients (Fig. 2) ${ }^{14)}$ Care was taken not to place the blade in the superior portion of the femoral neck in all cases.

\section{Radiological Assessment}

We compared the femoral neck-shaft angle of the injured side with the contralateral side in the radiographs taken immediately after the operation, and patients were divided into the valgus-reduced group (group 1) and the nonvalgus reduced group (group 2). The "valgus reduced" was defined as valgus reduction over $5^{\circ}$. In cases where contralateral proximal femoral fracture operation was also performed and it was difficult to check the neck-shaft angle of the contralateral side, any radiography performed in a previous study was used to check the neck-shaft angle on the contralateral side. Pelvis anteroposterior plain radiographs were taken at $10^{\circ}$ internal rotation with half of the lesser trochanter shown.

To measure how far down the blade tip was located in the femoral head, the calTAD was measured on pelvis anteroposterior and lateral plain radiographs taken imme-

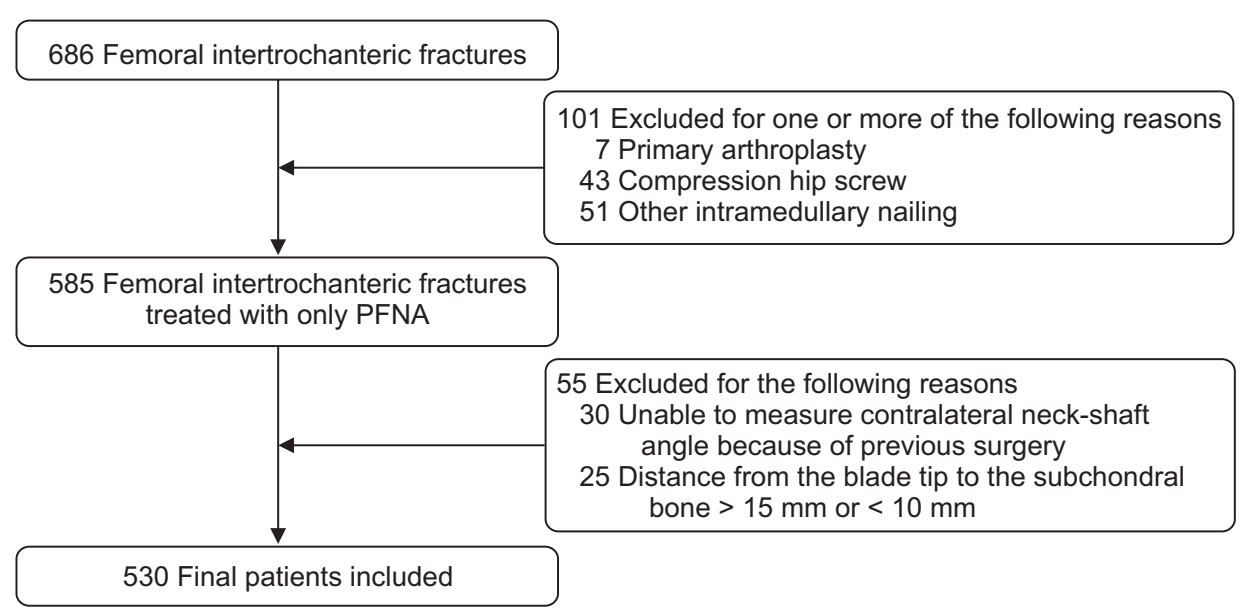

Fig. 1. The flowchart shows patient selection and exclusion criteria. PFNA: proximal femoral nail antirotation. 
diately after the operation (Fig. 3). ${ }^{10,15)}$

In addition, in order to determine how close the position of the blade to the medial femoral neck, the area between the blade and the medial femoral neck was calculated. On simple radiographs taken immediately after the operation, two inflection points where the femoral neck and the femoral head meet were connected by a line. Then, the line was moved in parallel to pass the inflection point where the femoral neck and the greater trochanter meet. The area of trapezoidal shape between the blade and the medial femoral neck was created. To correct for differences in the femoral neck length, the area was divided by the square of the height of the trapezoid (Fig. 4). The picture archiving and communication system (PACS; Marosis M-view 5.4, Marotech, Korea) was used for all measure- ments.

\section{Statistical Analysis}

The valgus-reduced group (group 1) and the non-valgus reduced group (group 2) were set as independent variables, and the calTAD and the area between the blade and the medial femoral neck were set as dependent variables. It can be assumed that both dependent variables have a normality of more than 30 samples, and independent sample $t$-test was performed to determine whether there was a significant difference between the two groups. The difference between the two groups was judged to be significant when the $p$-value was less than 0.05 . The statistical analysis was performed using IBM SPSS ver. 24.0 (IBM Corp., Armonk, NY, USA).
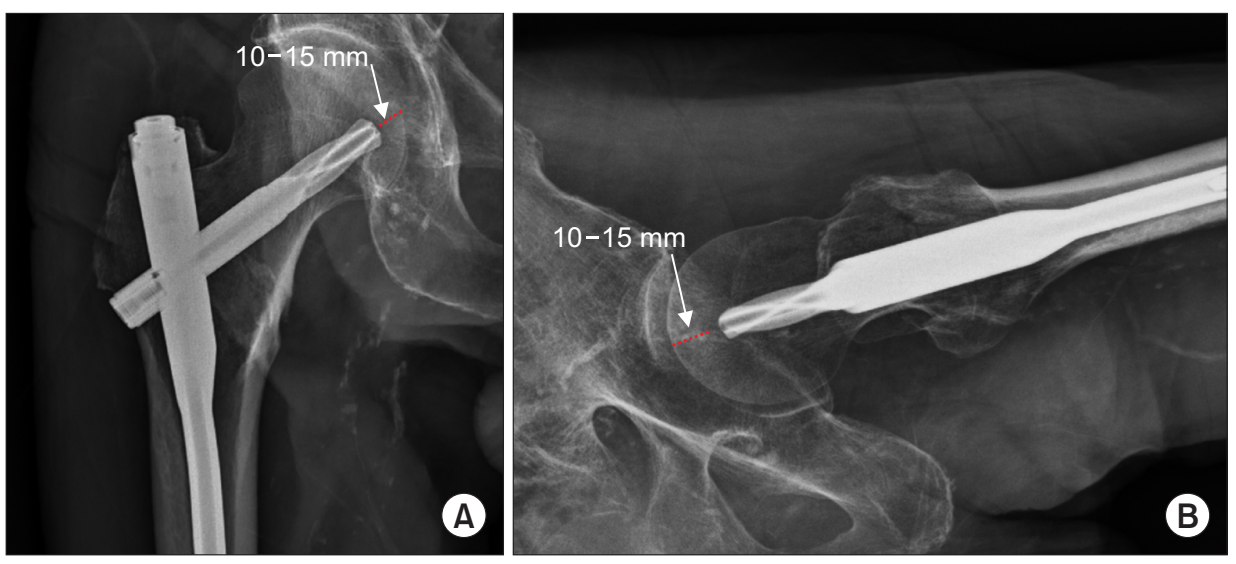

Fig. 2. The postoperative anteroposterior (A) and lateral (B) plain radiographs showed that the position of the blade tip was located within 10-15 $\mathrm{mm}$ of the subchondral bone.
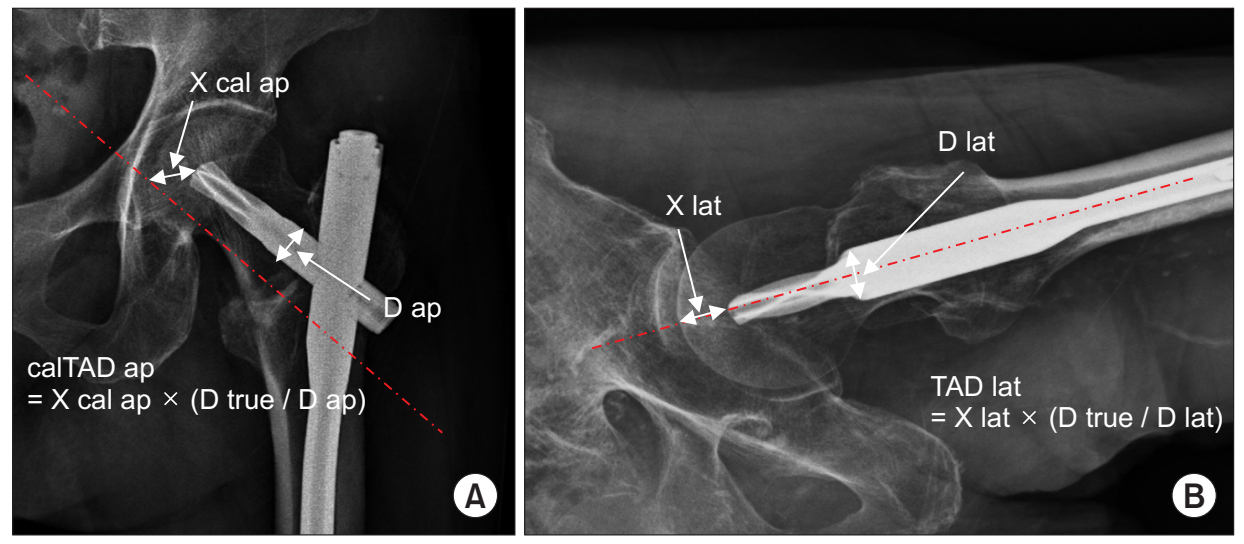

Fig. 3. Postoperative anteroposterior and lateral plain radiographs showing how to calculate the calcar referenced tip-apex distance (calTAD). (A) Tipapex distance (TAD) in reference to the calcar calculated on the anteroposterior radiograph (calTAD ap). Dotted line: the line passing through the midline of the femoral head and femoral neck was moved parallel to the medial femoral neck on the anteroposterior radiograph. (B) TAD calculated on the lateral radiograph (TAD lat). calTAD = calTAD ap + TAD lat. Dotted line: a line passing through the midline of the femoral head and femoral neck on the lateral radiograph. $X$ cal ap: the distance between the blade tip and the point where the dotted line and femoral head cortex meet on the anteroposterior radiograph, $\mathrm{D}$ ap: the calculated diameter of the lag-screw on the anteroposterior radiograph, $\mathrm{D}$ true: the known diameter of the lag-screw (10.5 mm), D lat: the calculated diameter of the lag-screw on the lateral radiograph, X lat: the distance between the blade tip and the point where the dotted line and femoral head cortex meet on the lateral radiograph. 

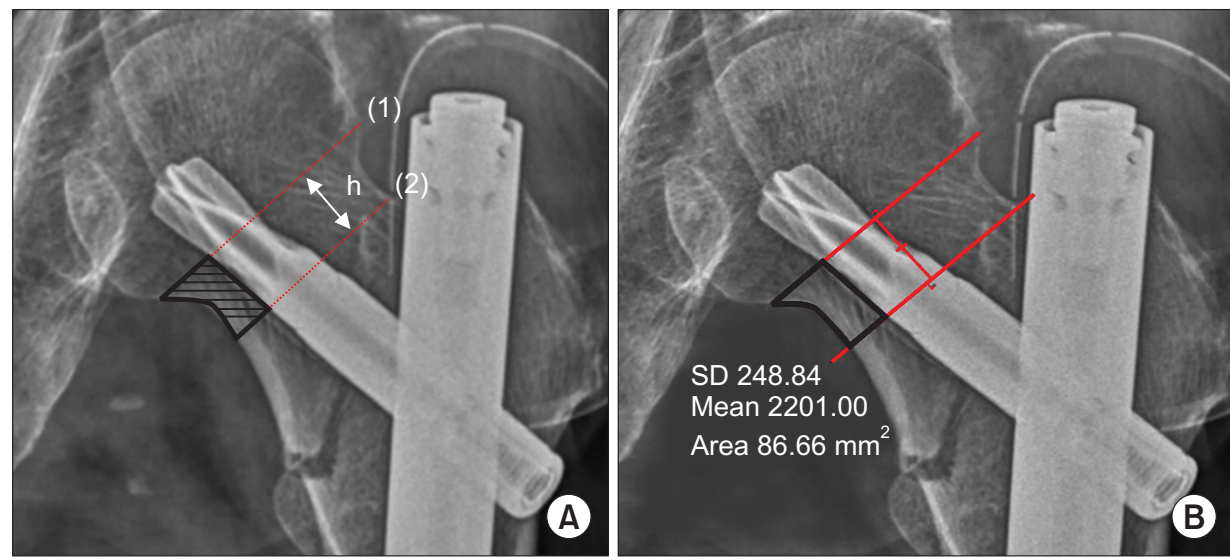

Fig. 4. Postoperative anteroposterior plain radiographs showing how to calculate the area between the blade and the medial femoral neck. (A) First, two inflection points where the femoral neck and the femoral head meet are connected by a line (1). Then, the line is moved in parallel to pass the inflection point where the femoral neck and the greater trochanter meet (2). The area of trapezoidal shape between the blade and the medial femoral neck is obtained. To correct for differences in the femoral neck length, the area is divided by the square of the height ( $h$ ) of the trapezoid. (B) Actual calculation process of the trapezoidal area on the picture archiving and communication system (PACS; Marosis M-view 5.4, Marotech, Seoul, Korea). SD: standard deviation.

\section{Table 1. Patient Demographics}

\begin{tabular}{|c|c|c|c|}
\hline Variable & Valgus reduced group $(n=416)$ & Non-valgus reduced group $(n=114)$ & $p$-value \\
\hline Sex (male:female) & $94: 322$ & $39: 75$ & $<0.05^{*}$ \\
\hline Age (yr) & $79.0 \pm 10.1$ & $77.6 \pm 12.0$ & $0.21^{\dagger}$ \\
\hline Implant type & & & $<0.05^{\dagger}$ \\
\hline PFNA $125^{\circ}$ & 117 & 55 & \\
\hline PFNA $130^{\circ}$ & 299 & 59 & \\
\hline Distance from blade tip to subchondral bone (mm) & $11.9 \pm 4.6$ & $12.2 \pm 4.6$ & $0.48^{*}$ \\
\hline
\end{tabular}

Values are presented as mean \pm standard deviation. Statistical significance was considered when $p$-values were less than 0.05 .

PFNA: proximal femoral nail antirotation.

${ }^{*}$ Chi-square test. ${ }^{\dagger}$ Independent $t$-test.

\section{RESULTS}

\section{Preoperative Evaluation}

Female patients were dominant in both group 1 (male:female, 94:322) and group 2 (male:female, 39:75). The mean age was $79.0 \pm 10.1$ years in group 1 and 77.6 \pm 12.0 years in group 2 . Both groups used $130^{\circ}$ PFNA implants more than $125^{\circ}$ PFNA implants. Preoperative demographic data are summarized in Table 1.

\section{Radiological Outcomes}

The valgus reduced group (group 1) had 418 cases and the non-valgus reduced group (group 2) had 112 cases. In group 1 , the mean valgus reduction was $8.7^{\circ} \pm 6.5^{\circ}$ and in group 2 , the value was $1.7^{\circ} \pm 1.8^{\circ}$. The calTAD was measured as $22.5 \pm 4.1 \mathrm{~mm}$ in group 1 and $24.9 \pm 3.7 \mathrm{~mm}$ in group $2(p<0.05)$. The area between the blade and the medial femoral neck was measured as $135.5 \pm 49.8 \mathrm{~mm}^{2}$ in group 1 and $145.0 \pm 55.1 \mathrm{~mm}^{2}$ in group 2 . The correction of the difference in the femoral neck length was $0.55 \pm 0.16$ in group 1 and $0.79 \pm 0.19$ in group $2(p<0.05)$ (Table 2 ).

\section{DISCUSSION}

The principal finding of this study is that in the valgusreduced group, the calTAD was decreased and the blade position was located inferiorly in the femoral neck, resulting in firmer fixation at the femoral head and neck. Treat- 
Table 2. Intergroup Comparison of Results

\begin{tabular}{|c|c|c|c|}
\hline Variable & $\begin{array}{l}\text { Valgus reduced group } \\
\qquad(\mathrm{n}=416)\end{array}$ & $\begin{array}{l}\text { Non-valgus reduced group } \\
\qquad(\mathrm{n}=114)\end{array}$ & $p$-value ${ }^{*}$ \\
\hline Valgus reduction $\left({ }^{\circ}\right)$ & $8.7 \pm 6.5$ & $1.7 \pm 1.8$ & $<0.05$ \\
\hline calTAD (mm) & $22.5 \pm 4.1$ & $24.8 \pm 3.8$ & $<0.05$ \\
\hline $\begin{array}{l}\text { Area between the blade and the medial femoral neck } \\
\text { (area of trapezoid, } \mathrm{mm}^{2} \text { ) }\end{array}$ & $135.5 \pm 49.8$ & $145.1 \pm 54.8$ & 0.074 \\
\hline Area of trapezoid/square of height of trapezoid & $0.6 \pm 0.2$ & $0.8 \pm 0.2$ & $<0.05$ \\
\hline
\end{tabular}

Values are presented as mean \pm standard deviation. Statistical significance was considered when $p$-values were below 0.05 . calTAD: calcar referenced tip-apex distance.

*Independent $t$-test.
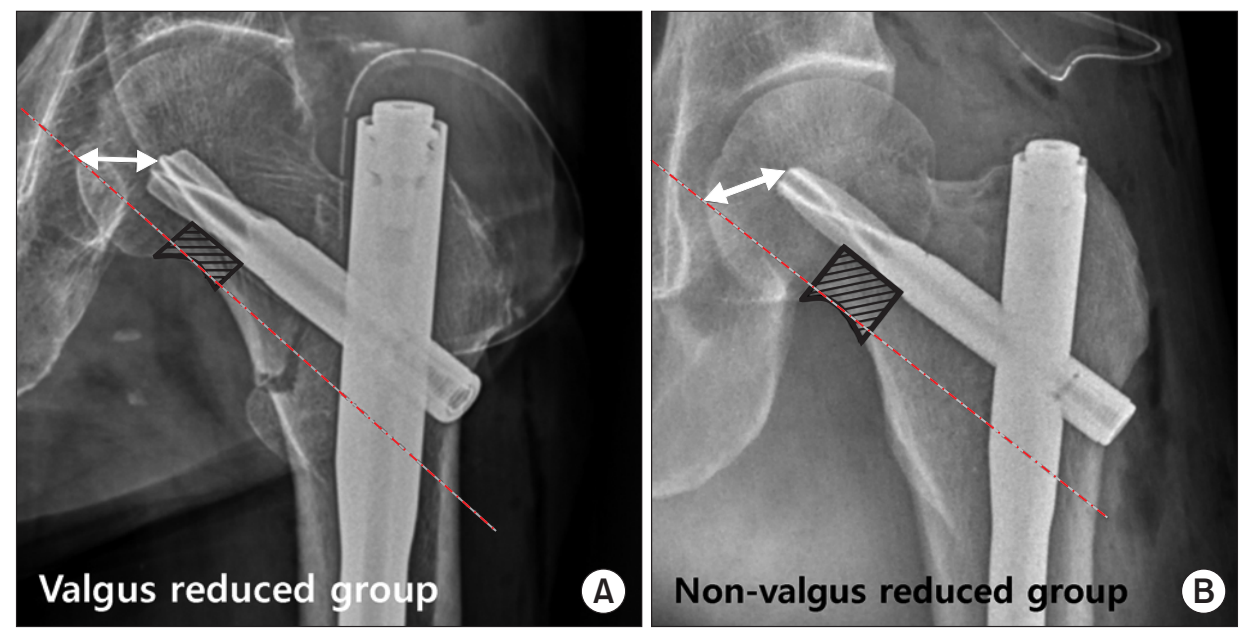

Fig. 5. Comparison of the valgus reduced group $(A)$ and the non-valgus reduced group (B). In the valgus reduced group, the calcar referenced tip-apex distance and the area between the blade and the medial femoral neck are smaller than those in the non-valgus group. In other words, in the valgus-reduced group, the blade position was located inferiorly in the femoral neck.

ment of intertrochanteric hip fractures is important to improve the prognosis in patients with early ambulation. Currently, internal fixation with PFNA and several valgus reduction techniques have been widely used to decrease the risk of varus deformity due to flexion moment that occur during early gait. Laskin et al. ${ }^{16)}$ reported satisfactory results using the Wayne-County reduction method, which is a valgus reduction method that overlaps the medial and posterior cortices of the proximal femur in unstable intertrochanteric hip fractures with posteromedial bone defect. Choi et al. ${ }^{6}$ ) also reported that the Wayne-County reduction method was superior to the anatomic reduction method in terms of radiographic and clinical outcomes in unstable intertrochanteric hip fractures. Chang et al. ${ }^{17)}$ reported that radiologically excellent results were obtained using positive medial cortical support, which is a nonanatomic reduction method that locates the medial cortex of proximal fragment superomedial to the medial cortex of distal fragment. Park et al. ${ }^{7)}$ and Jiamton et al. ${ }^{18)}$ also reported that valgus reduction was an effective treatment to decrease the failure of internal fixation, especially in unstable intertrochanteric hip fractures and to obtain good radiological and clinical results.

When PFNA is used, the blade may cause major complications such as perforation of the femoral head, so the positioning of the blade is very important. Jiamton et al. ${ }^{18)}$ reported that the risk of perforation of the femoral head was decreased when the tip of the blade was located in the inferior half of the femoral head. Kashigar et al., ${ }^{10)}$ Caruso et al., ${ }^{15)}$ and Puthezhath and Jayaprakash ${ }^{12)}$ reported that the risk was decreased when the calTAD decreased. In addition, according to Stiehl et al.," ${ }^{9)}$ the inferior side of the femoral neck is a dense trabecular structure, which is more resistant to loading stress than other parts of the femoral neck. Therefore, positioning the blade at the inferior half of the femoral head and the inferior side of the femoral neck during internal fixation is advantageous for obtaining stable fixation. However, there was no study on the relationship between the status of reduction and the position of the blade of the PFNA. Therefore, this study 
has significance as the first study to quantify the relationship between the two.

In this study, 418 of 530 cases were group 1 and 112 cases were group 2 . The calTAD was significantly smaller in group 1 than in group 2, indicating that valgus reduction is advantageous for positioning the blade tip in the inferior half of the femoral head. There is no quantification method available for proximity of the blade to the medial femoral neck, so we measured the area between the blade and the medial femoral neck under the assumption that the blade was located near the medial femoral neck when the area was small. In group 1, the area was smaller than that in group 2, indicating that the blade was located closer to the medial femoral neck and the firm fixation force could be obtained (Fig. 5).

There are limitations of this study. First, there may be bias inherent to the retrospective design. To overcome this limitation, we measured the calTAD and the area be- tween the blade and the medial femoral neck in a blinded manner before groups. Second, we did not investigate the clinical outcome. In previous papers, the impact of blade position on the clinical outcome was already addressed. ${ }^{10,12,15,18)}$ Therefore, we focused on assessing the quality of reduction and the position of the blade radiologically in this study. We think that it will be also meaningful to follow the clinical outcomes of each group. Valgus reduction resulted in less calTAD and inferior position of the blade at the femoral neck in the treatment of intertrochanteric hip fractures with PFNA. In addition, it seems to provide firm fixation at the femoral head and neck.

\section{CONFLICT OF INTEREST}

No potential conflict of interest relevant to this article was reported.

\section{REFERENCES}

1. Richmond J, Aharonoff GB, Zuckerman JD, Koval KJ. Mortality risk after hip fracture. J Orthop Trauma. 2003;17(1):53-6.

2. Zhang K, Zhang S, Yang J, et al. Proximal femoral nail vs. dynamic hip screw in treatment of intertrochanteric fractures: a meta-analysis. Med Sci Monit. 2014;20:1628-33.

3. Kaufer H, Matthews LS, Sonstegard D. Stable fixation of intertrochanteric fractures. J Bone Joint Surg Am. 1974;56(5):899-907.

4. Ma KL, Wang X, Luan FJ, et al. Proximal femoral nails antirotation, Gamma nails, and dynamic hip screws for fixation of intertrochanteric fractures of femur: a meta-analysis. Orthop Traumatol Surg Res. 2014;100(8):859-66.

5. Chang SM, Zhang YQ, Du SC, et al. Anteromedial cortical support reduction in unstable pertrochanteric fractures: a comparison of intra-operative fluoroscopy and post-operative three dimensional computerised tomography reconstruction. Int Orthop. 2018;42(1):183-9.

6. Choi NY, Nah KH, Song HS, Seo SI, Choi JK, Han SK. Treatment of the intertrochanteric fractures of the femur in elderly patients: comparision of Wayne-County reduction and anatomical reduction. J Korean Fract Soc. 2004;17(4):301-7.

7. Park JK, Shon HC, Kim YM, et al. Effectiveness of the valgus reduction technique in treatment of intertrochanteric fractures using proximal femoral nail antirotation. J Korean Orthop Assoc. 2013;48(6):441-8.

8. Lee DY, Hwang SC, Jeong ST, Oh JY, Kim DH. Risk fac- tors of cut-out in treatment of femoral intertrochanteric fractures by proximal femur nail. J Korean Orthop Assoc. 2017;52(4):319-26.

9. Stiehl JB, Jacobson D, Carrera G. Morphological analysis of the proximal femur using quantitative computed tomography. Int Orthop. 2007;31(3):287-92.

10. Kashigar A, Vincent A, Gunton MJ, Backstein D, Safir O, Kuzyk PR. Predictors of failure for cephalomedullary nailing of proximal femoral fractures. Bone Joint J. 2014;96(8):102934.

11. Paul O, Barker JU, Lane JM, Helfet DL, Lorich DG. Functional and radiographic outcomes of intertrochanteric hip fractures treated with calcar reduction, compression, and trochanteric entry nailing. J Orthop Trauma. 2012;26(3):148-54.

12. Puthezhath $\mathrm{K}$, Jayaprakash $\mathrm{C}$. Is calcar referenced tip-apex distance a better predicting factor for cutting out in biaxial cephalomedullary nails than tip-apex distance? J Orthop Surg (Hong Kong). 2017;25(3):2309499017727920.

13. Carr JB. The anterior and medial reduction of intertrochanteric fractures: a simple method to obtain a stable reduction. J Orthop Trauma. 2007;21(7):485-9.

14. Haidukewych GJ. Intertrochanteric fractures: ten tips to improve results. J Bone Joint Surg Am. 2009;91(3):712-9.

15. Caruso G, Bonomo M, Valpiani G, et al. A six-year retrospective analysis of cut-out risk predictors in cephalomedullary nailing for pertrochanteric fractures: can the tip-apex 
distance (TAD) still be considered the best parameter? Bone Joint Res. 2017;6(8):481-8.

16. Laskin RS, Gruber MA, Zimmerman AJ. Intertrochanteric fractures of the hip in the elderly: a retrospective analysis of 236 cases. Clin Orthop Relat Res. 1979;(141):188-95.

17. Chang SM, Zhang YQ, Ma Z, Li Q, Dargel J, Eysel P. Fracture reduction with positive medial cortical support: a key element in stability reconstruction for the unstable pertrochanteric hip fractures. Arch Orthop Trauma Surg. 2015;135(6):811-8.

18. Jiamton C, Boernert K, Babst R, Beeres FJ, Link BC. The nail-shaft-axis of the of proximal femoral nail antirotation (PFNA) is an important prognostic factor in the operative treatment of intertrochanteric fractures. Arch Orthop Trauma Surg. 2018;138(3):339-49. 